\title{
Mitochondrial encephalopathy with stroke-like episodes and lactate acidosis (MELAS syndrome): diagnostic criteria, features of epileptic seizures and treatment approaches
}

\begin{abstract}
The paper describes a patient with mitochondrial encephalopathy, lactic acidosis, and strokelike episodes syndrome (MELAS). The features of the course and therapy of epilepsy in MELAS are discussed. The disease is known for its late diagnosis when years elapse from the onset of the clinical manifestations to diagnosis. The paper presents clinical criteria for the diagnosis of MELAS and the specific features of brain neuroimaging changes that allow identification of the disease at an early stage.
\end{abstract}

Keywords: MELAS syndrome, criteria for diagnosis, neuroimaging signs, clinical case
Volume 9 Issue 5 - 2019

\author{
Yamin MA,' Belyaev OV, ${ }^{2}$ Araslanova LV' \\ 'SAU of the Rostov Region, Regional Consultative and \\ Diagnostic Center, Russia \\ 2Department of Neurology, Volgograd State Medical University, \\ Russia
}

Correspondence: OV Belyaev, Department of Neurology, Neurosurgery, Medical Genetics, with a course of neurology, manual therapy, reflexotherapy,Volgograd State Medical University,Volgograd, Russia, Email epileptic-center@mail.ru

Received: May 15, 2019 | Published: September 02, 2019
Abbreviations: MB, Mitochondrial diseases; mtDNA, mitochondrial DNA; MRI, magnetic resonance imaging; GTCS, generalized tonic-clonic seizure; HSV, herpes simplex virus; ENMG, electroneuromyography

\section{Introduction}

Mitochondrial diseases (MB) are a large heterogeneous group of diseases resulting from genetic mutations of nuclear or mitochondrial DNA (mtDNA) and manifested by impaired oxidative phosphorylation in mitochondria and energy production in cells. The leading neurological syndromes in MB are epileptic, cerebellar, myopathic, and polyneuritic. ${ }^{1}$

Epilepsy develops in about $40-60 \%$ of patients with MB. The most frequently described myoclonic attacks, marked in isolation or in combination with focal (motor), tonic, clonic and tonic-clonic ${ }^{2}$. Some of the antiepileptic drugs traditionally used in these types of attacks with MB can significantly aggravate the patient's condition ${ }^{3,}$ ${ }^{4}$. A variety of clinical symptoms, severity, features of therapy cause difficulties in establishing the diagnosis and management of such patients.

The main clinical criteria for the diagnosis of MELAS are:5-7
a. maternal type of inheritance;
b. age of occurrence up to 40years;
c. normal psychomotor development before the disease;
d. intolerance to physical exertion;
e. migraine headache with nausea and vomiting;
f. stroke-like episodes;
g. encephalopathy with epileptic seizures and/or dementia;

h. lactic acidosis;

i. torn red fibers in skeletal muscle biopsies;

j. progressive course.

We give the case history of a patient with MELAS syndrome. Patient A., born in 1990, came in April 2011 with complaints of hearing impairment in both ears, increased muscle fatigue, lethargy, periodic (2-3times) weekly, intense throbbing headache, more pronounced on the left, accompanied by nausea, duration before the day. Also disturbed by a slight precariousness when walking, simple visual hallucinations (colored balls, spots), lack of body weight. From March 30, 2011, vision began to deteriorate, and on April 4, vision was completely disturbed. The conclusion of the oculist: cortical amaurosis.

From the anamnesis it is known that the child was born from the first pregnancy in time, the birth was uneventful. Newborn period and early psychomotor development without deviation from the norm. Family history is not burdened, parents are not consanguineous.

He considers himself a patient since 2004 (from the age of 13), when, against the background of stress, his appetite was disturbed, leg edema, general weakness, auditory hallucinations appeared. He was treated with a diagnosis of obsessive-compulsive disorder. For 6 months he received trifluoperazine, lithium carbonate, amitriptyline. Magnetic resonance imaging (MRI), performed in 2004, revealed a single lesion on the right in the projection of the posterior femur of the inner capsule.

According to the father, from 2005 to 2009 , adolescents had increased fatigue, leg edema, and body mass deficiency. On March 2, 2009, catarrhal symptoms (cough) and nosebleeds appeared on the background of an increase in body temperature to $39 \mathrm{C}$. He was treated on an outpatient basis. On March 10, 2009, hearing deteriorated 
sharply (it was especially difficult to distinguish speech), auditory hallucinations and abundant salivation appeared. During his stay in the hospital, he was worried by a severe headache of migraine nature, simple visual hallucinations in the form of colored balls, spots. March 15th developed a generalized tonic-clonic seizure (GTCS). Performed MRI of the brain (Figure 1).

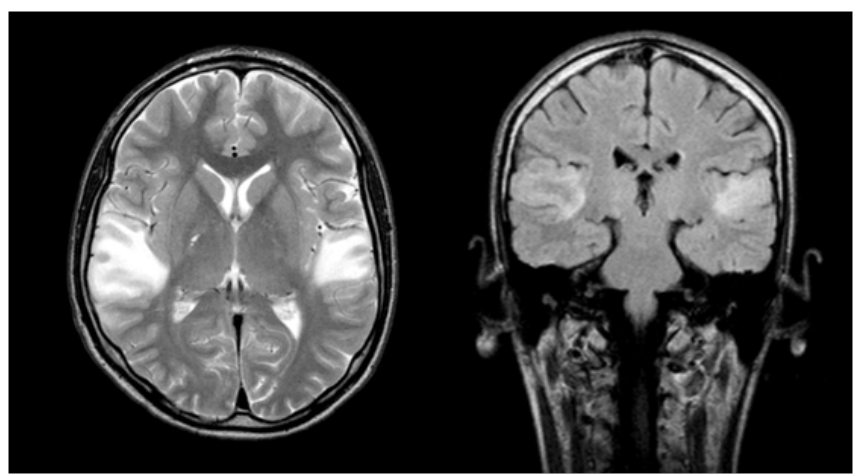

Figure I Brain MRI in T2VI and FLAIR modes (March 2009). Symmetric foci of increased MR signal intensity in the cortico-subcortical portions of the temporal lobes of both hemispheres are determined.

A spinal puncture was performed: the cerebrospinal fluid is colorless, transparent, protein $-0.099 \mathrm{~g} / 1$, cytosis -7 cells per $1 \mu \mathrm{l}$ (lymphocytes $-100 \%$ ), glucose $-2.0 \mathrm{mmol} / 1$, chlorides $-118 \mathrm{mmol} / 1$. In the study of cerebrospinal fluid by the method of polymerase chain reaction revealed herpes simplex virus (HSV) type 1-2. The data of enzyme immunoassay on CSV type 1-2, cytomegalovirus, Epstein - Barr virus are negative. Diagnosed: viral encephalitis herpetic etiology. During therapy (medovir, mexiprim, actovegin, reamberin, group B vitamins, cytoflavin, cyfran, valproic acid, pagluferal), the patient's condition improved (seizures were relieved, headache decreased, hearing recovered, and general well-being improved).

After discharge, he continued to take valproic acid $150 \mathrm{mg}$ and pagluferal 1 tablet per day, against this background night-time (at 3-4 am) persisted GTEC with a frequency of up to 1 time in 3months, focal non-motor attacks (visual hallucinations, impaired consciousness) up to $4-5$ times a day.

In November 2009, the state of health worsened: frequent attacks of freezing, GTCS, on this background developed rightsided hemianopsia, right-sided pyramidal insufficiency and hemihypesthesia. He was hospitalized with a diagnosis of acute cerebrovascular accident in the basin of the left posterior cerebral artery of November 28, 2009 with right-sided hemianopia and pyramidal insufficiency. Mitochondrial encephalomyopathy?

During therapy (Actovegin, group B vitamins, cytoflavin, emoxipin, a polarizing mixture), the patient's condition improved significantly. After discharge, blood lactate and pyruvate levels, as well as electroneuromyography (ENMG), were recommended. The dose of valproic acid is increased to $300 \mathrm{mg} /$ day, it is recommended to take Kudesan.

Subsequent hospitalizations in March and October 2010 were due to an increase in epileptic seizures, both focal dis- cognitive and secondary generalized tonic-clonic, as well as intense daily headache, more pronounced in the morning. According to the extracts, there were attacks of increased headache, which were accompanied by nausea, retching, severe general weakness and increased blood pressure, skin hyperemia. The duration of attacks - up to 5 hours, after which the patient has been slowed down for a long time, drowsy.
During hospitalization in October 2010, a medical conference was held and a diagnosis was made: chronic herpetic recurrent encephalitis, which must be differentiated from MELAS syndrome (Figure 2).

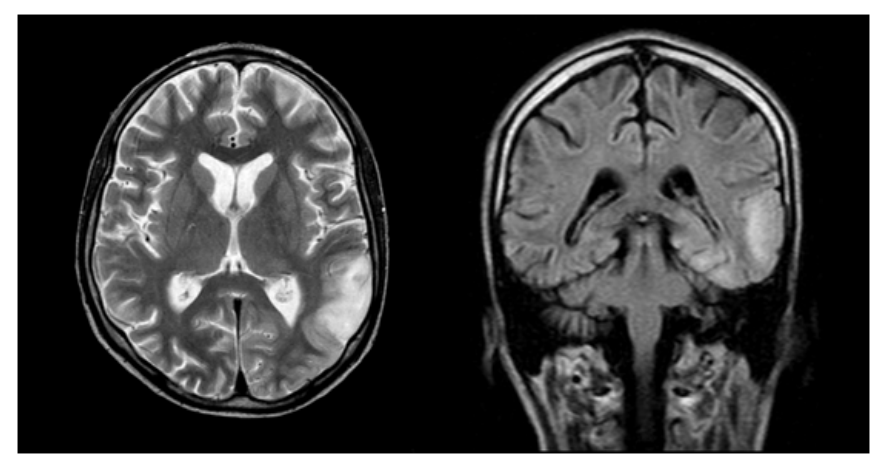

Figure $2 \mathrm{MRI}$ of the brain in T2 VI and FLAIR modes (September 2010). One can see a center of increased intensity of the MR signal in the corticosubcortical regions of the left parietal and occipital lobes, a regression of the focus in the right temporal lobe.

The patient's condition worsened, there were attacks of the type of right-sided hemiconvulsions, episodes of visual impairment lasting from 1 to 5hours, simple visual hallucinations, stopped by intramuscular administration of diazepam (Figure 3) were observed. Since February 2010, he received valproic acid $1500 \mathrm{mg} /$ day, $3 / 4$ pagluferal tablets.

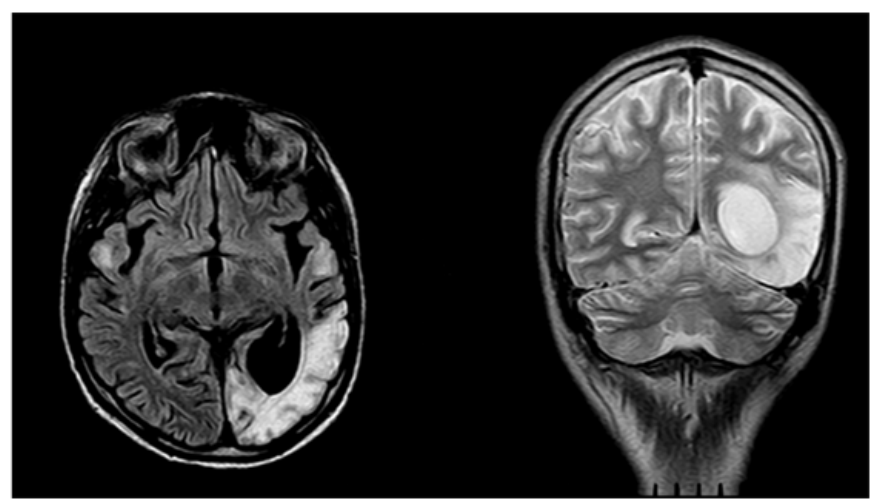

Figure $3 \mathrm{MRI}$ of the brain in FLAIR and T2 VI modes (February 20II). There is a focus of increased signal intensity in the cortico-subcortical regions of the left occipital lobe.

When examining a patient of reduced nutrition (body weight $39 \mathrm{~kg}$ with height $168 \mathrm{~cm}$; Figure 4). Neurological status: vision at the level of light perception; bilateral hypoacusia. Deep reflexes are reduced, positive Babinsky symptom from two sides.
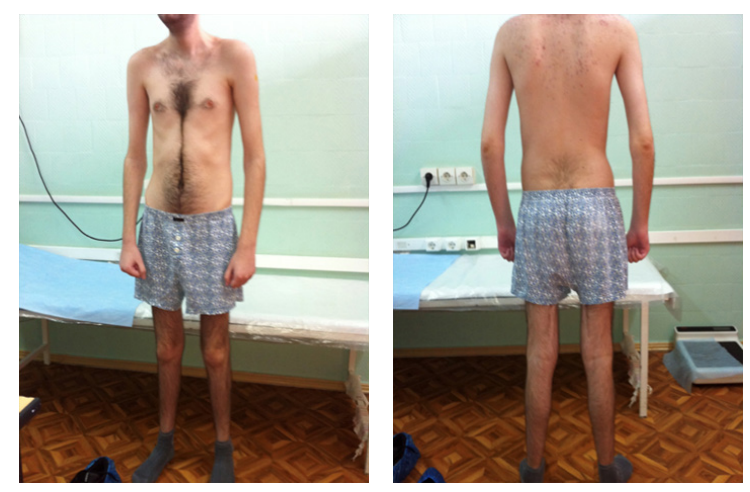

Figure 4 Patient A April 20II. 
The blood lactate level is $5.78 \mathrm{mmol} / 1(0.50-2.00), \mathrm{pH}$ is 7.337 (7.350-7.450). When ENMG revealed changes characteristic of the primary muscle type, and for axonal pathology. In electroencephalography (EEG), the slowing down of the main activity of the background recording compared with the age norm, expressed diffuse disturbances of the bioelectrical activity of the brain were noted. Typical epileptiform activity is not registered (Figure 5).

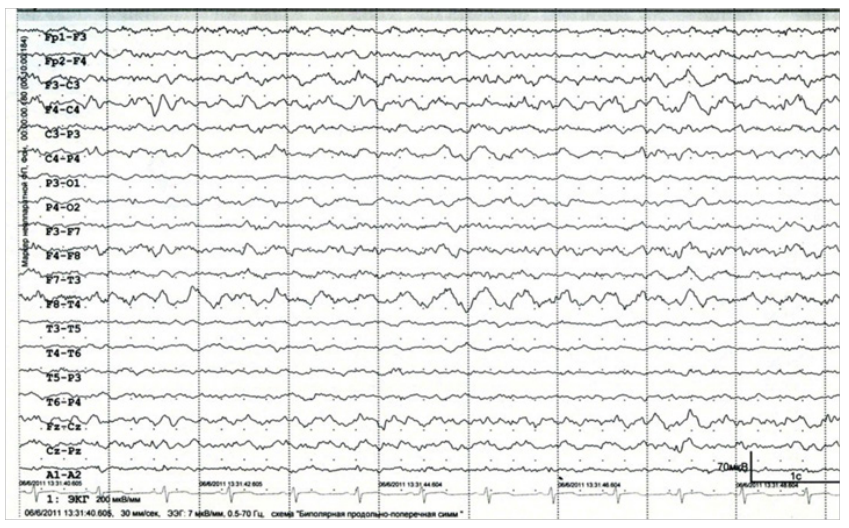

Figure 5 EEG (April 20II). There are marked diffuse changes in the bioelectrical activity of the brain, slowing down the main activity of the background recording in comparison with the age norm.

DNA diagnostics was carried out: the A3243G mutation characteristic of the MELAS syndrome was determined. Treatment prescribed: noben $90 \mathrm{mg}$, carnitetin 3 capsules and levetiracetam $1500 \mathrm{mg}$ per day, slow withdrawal of pagluferal and valproic acid is recommended. In the course of treatment, vision and hearing improved, fatigue decreased, epileptic seizures and migraine-like headache were stopped, and overall well-being improved.

The patient's condition remained stable only for a year. Since April 2012, my eyesight has deteriorated again. MRI of the brain (October 2012) showed a massive scar-atrophic process in both occipital lobes, cortico-subcortical foci of gliosis in the temporal lobes (Figure 6).

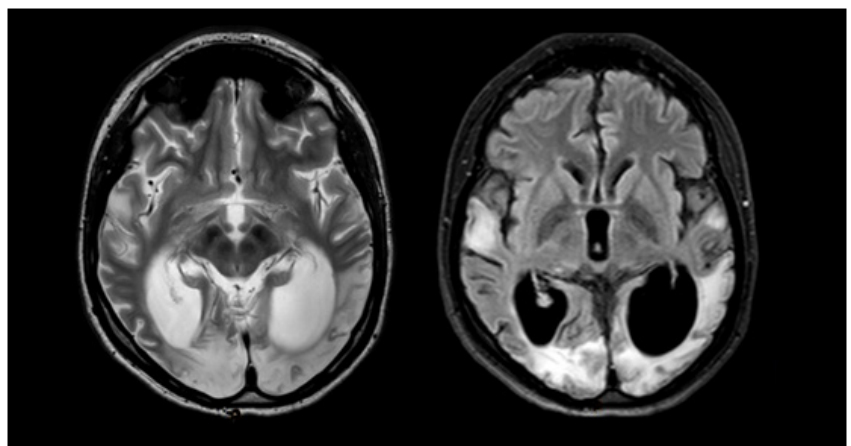

Figure 6 MRI of the brain in T2 VI and FLAIR modes (October 2012). A massive cicatricial-atrophic process in both occipital lobes is visualized. Cortico-subcortical foci of gliosis in the temporal lobes.

In early December 2012, weakness in the neck muscles, impaired coordination of movements, myoclonic seizures, stopped eating, and the level of consciousness decreased. December 7, examined at home: for contact is not available, there was a constant myoclonus in the muscles of the neck, arms, abdomen, weakness of the muscles of the neck (does not hold the head). An ambulance crew delivered a hospital, diagnosis: myoclonic status epilepticus. EEG registered myoclonic status (generalized poly-wave activity; Figure 7). On the night of December 9, 2012 death occurred.

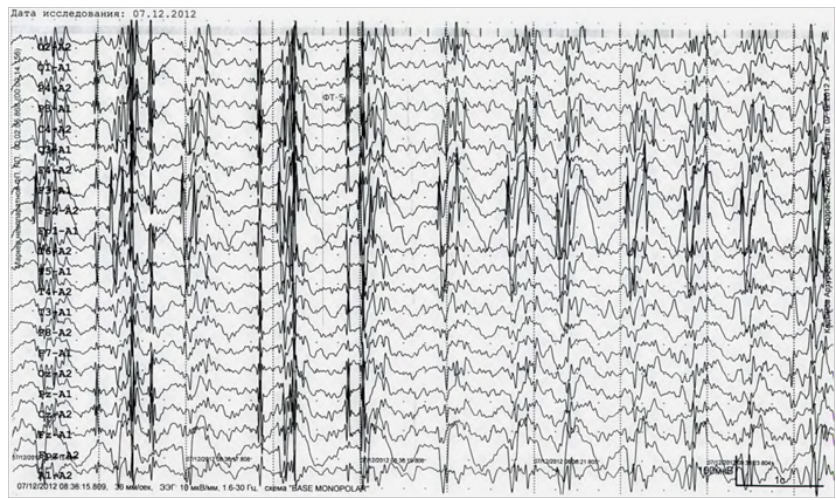

Figure 7 EEG (December 7, 20I2). Myoclonic status: generalized polywave activity.

\section{Discussion}

Within the framework of this article it is impossible to discuss all the features of mitochondrial diseases, therefore we will focus on the most important ones. First, mitochondrial genetics has three significant differences from Mendelian one: 1) maternal inheritance — the child receives the entire cytoplasm with organelles in it along with the egg, that is, only from the mother; 2) heteroplasmy is a condition in which mitochondria coexist in the cytoplasm of a cell with two different variants of mtDNA (normal and mutant), unlike homoplasmy, in which the mtDNA of all mitochondria of a cell is represented by one type (mutant or normal); 3) mitotic segregation - an uneven, random separation of cell organelles during mitosis. In the process of formation of primary germ cells, a series of consecutive divisions leads to the fact that in the formed cells the ratio of mitochondria with mutant and normal mtDNA will differ significantly from their ratio in the original (mother) cell, i.e., a different level of heteroplasmy will be observed. The further process of maturation of the primary germ cells to the extent of the egg cell is characterized only by an increase in the number of organelles (mitochondria), but the level of heteroplasmia does not change (Figure 8).

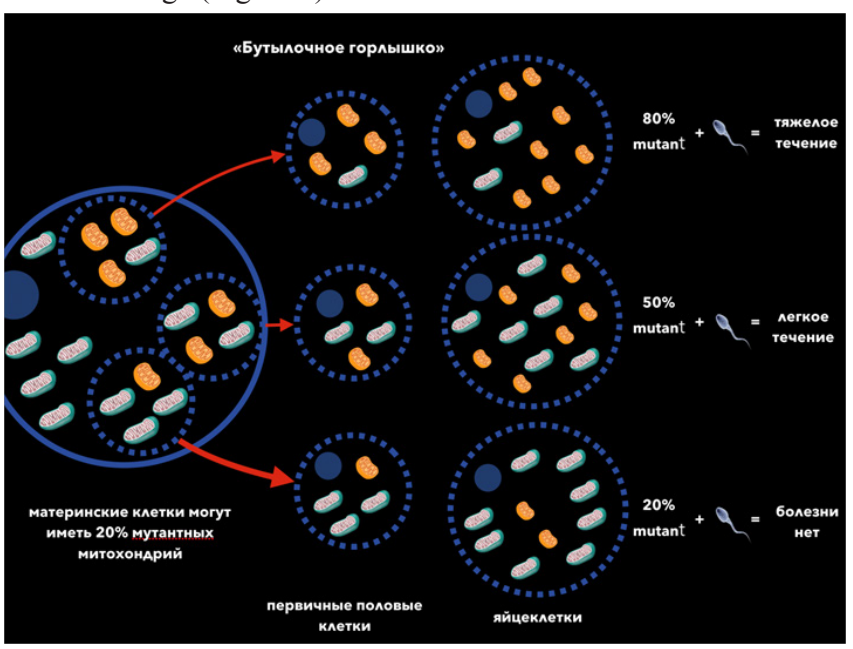

Figure 8 The level of heteroplasmy determines the severity of MB. For the manifestation of the disease, it is necessary that the number of mutant mtDNA exceeds a certain level - this phenomenon is called threshold effect. ${ }^{8}$

"Bottleneck" $80 \%$ mutant $+=$ severe course

$50 \%$ mutant $+=$ mild for $20 \%$ mutant $+=$ no disease 
Oocytes Primary germ cells Mother cells can have $20 \%$ of mutant mitochondria

Second, although about $15 \mathrm{mtDNA}$ mutations are associated with MELAS syndrome, approximately $80-90 \%$ is a mutation $3243 \mathrm{~A}>\mathrm{G}$. therefore the initial screening should be directed specifically at this mutation. ${ }^{9}$

Thirdly, stroke-like episodes in MELAS ${ }^{10}$ have the following features: 1) young age (usually up to 40years old); 2) frequent presence of a provoking factor (occur after febrile temperature, an epileptic seizure, migraine-like headache); 3 ) the favorite localization is the occipital region; 4) foci, as a rule, are outside the area of large cerebral arteries, often affecting the cortex or deep structures of the white matter of the brain.

Fourthly, in MELAS syndrome, myoclonic seizures are most frequently recorded, but focal sensory, motor, and secondary generalized tonic-clonic seizures are also observed. ${ }^{5}$

In our patient, 7years have passed since the onset of symptoms until the diagnosis was established. What makes it possible to suspect mitochondrial disease at an earlier stage? First of all, it is a combination of a certain phenotype (marked lack of body weight, poor exercise tolerance) and the clinical picture (stroke-like episodes at a young age against the background of such provoking factors as stress, febrile temperature, etc., migraine-like headache, epileptic seizures, progressive course ). MRI of the brain (favorite localization of foci in the temporal and occipital regions) and laboratory tests (elevated blood lactate and CSF) provide additional information.

\section{Treatment}

Currently, there is no effective pathogenetic treatment of MELAS syndrome, symptomatic treatment is carried out. One of the most promising treatments for MELAS syndrome is the use of L-arginine. During an acute stroke-like episode, intravenous bolus of L-arginine $\left(500 \mathrm{mg} / \mathrm{kg}\right.$ for children or $10 \mathrm{~g} / \mathrm{m}^{2}$ of body area for adults) for 3 hours after the onset of symptoms can quickly reduce the severity of the condition by reducing lactate levels and improving microcirculation. After the initial bolus, continuous infusion is continued for 24hours at a similar dose of 3-5days. A further transition to oral administration of the drug at a dose of $0.15-0.3 \mathrm{~g} / \mathrm{kg} /$ day reduces the frequency and severity of stroke-like episodes. ${ }^{11,12}$ However, it must be borne in mind that an overdose of L-arginine can cause hypotension and severe hyponatremia (possibly due to natriuresis due to increased nitric oxide production), as well as the development of central pontin / extrapontin myelinolysis with the rapid correction of hyponatremia. ${ }^{13}$ In recent years, data on the use of L-citrulline in patients with MELAS. It is a precursor of L-arginine, but, unlike the latter, it increases the production of nitric oxide to a greater extent due to a significant increase in de novo synthesis of arginine. ${ }^{14,15}$

Also use drugs that promote electron transfer in the respiratory chain (coenzyme Q10, idebenone, vitamins K1 and K3, succinic acid, cytochrome $\mathrm{C}$, ascorbic acid), activating beta-oxidation (L-carnitine), linking the active oxygen species (lipoic acid), being alternative energy sources (creatine monohydrate). ${ }^{16-18}$

Previously prescribed drugs that reduce the level of lactate (dichloroacetate, dimephosphone) are not currently used, as they can enhance polyneuropathy. ${ }^{19}$ In the treatment of epileptic seizures, valproic acid and phenobarbital are not recommended. ${ }^{3,4,20-22}$ The drugs of choice are oxcarbazepine, topiramate, levetiracetam.

\section{Acknowledgments}

None.

\section{Conflicts of interest}

The authors declare there are no conflicts of interest related to the article.

\section{References}

1. Haas RH, Parikh S, Falk MJ, et al. Mitochondrial disease: a practical approach for primary care physicians. Pediatrics. 2007;120(6):1326-1333.

2. Darin N, Oldfors A, Moslemi AR, et al. The incidence of mitochondrial encephalomyopathies in childhood: clinical features and morphological, biochemical, and DNA anbormalities. Ann Neurol. 2001;49(3):377-383.

3. Lam CW, Lau CH, Williams JC, et al. Mitochondrial myopathy, encephalopathy, lactic acidosis and stroke-like episodes (MELAS) triggered by valproate therapy. Eur J Pediatr. 1997;156(7):562-564.

4. Chaudhry N, Patidar Y, Puri V. Mitochondrial myopathy, encephalopathy, lactic acidosis, and stroke-like episodes unveiled by valproate. $J$ Pediatr Neurosci. 2013;8(2):135-137.

5. Pavlakis SG, Phillips PC, Di Mauro S, et al. Mitochondrial myopathy, encephalopathy, lactic acidosis, and stroke-like episodes: a distinctive clinical syndrome. Ann Neurol. 1984;16:481-488.

6. Hirano M, Ricci E, Koenigsberger MR, et al. Melas: an original case and clinical criteria for diagnosis. Neuromuscular Disord. 1992;2(2):125-135.

7. Hirano M, Pavlakis SG. Mitochondrial myopathy, encephalopathy, lactic acidosis, and stroke like episodes (MELAS): current concepts. J Child Neurol. 1994; 9(1): 4-13.

8. Mazunin IO, Volod'ko NV, Starikovskaia EB, et al. Mitochondrial genome and human mitochondrial diseases. Molecular Biology. 2010;44(5):755772 .

9. Ciafaloni E, Ricci E, Shanske S, et al. MELAS: clinical features, biochemistry, and molecular genetics. Ann Neurol. 1992;31(4):391-398.

10. Matthews PM, Tampieri D, Berkovic SF, et al. Magnetic resonance imaging shows specific abnormalities in the MELAS syndrome. Neurology. 1991;41(7):1043-1046.

11. Koenig MK, Emrick L, Karaa A, et al. Recommendations for the management of strokelike episodes in patients with mitochondrial encephalomyopathy, lactic acidosis, and strokelike episodes. JAMA Neurol. 2016;73(5):591-594.

12. Koga Y, Akita Y, Nishioka J, et al. L-arginine improves the symptoms of stroke-like episodes in MELAS. Neurology. 2005;64(4):710-712.

13. Koga Y, PovalkoN, Nishioka J, et al. MELAS and L-arginine therapy: pathophysiology of stroke-like episodes. Ann N Y Acad Sci. 2010;1201:104-110.

14. El-Hattab AW, Adesina AM, Jones J, et al. MELAS syndrome: clinical manifestations, pathogenesis, and treatment options. Mol Genet Metab. 2015;116(1-2):4-12.

15. El-Hattab AW, Almannai M, Scaglia F. Arginine and citrulline for the treatment of MELAS syndrome. J Inborn Errors Metab Screen. 2017:5.

16. Rodriguez MC, MacDonald JR, Mahoney DJ, et al. Benecial effects of creatine, CoQ10, and lipoic acid in mitochondrial disorders. Muscle Nerve. 2007;35(2):235-242.

17. Napolitano A, Salvetti S, Vista M, et al. Long-term treatment with idebenone and riboflavin in a patient with MELAS. Neurol Sci. 2000;21(5 Suppl):S981-S582. 
18. Pfeffer G, Majamaa K, Turnbull DM, et al. Treatment for mitochondrial disorders. Cochrane Database Syst Rev. 2012;4:CD004426.

19. Kaufmann P, Engelstad K, Wei Y, et al. Dichloroacetate causes toxic neuropathy in MELAS: a randomized, controlled clinical trial. Neurology. 2006;66(3):324-330.

20. Luis PB, Ruiter JP, Aires CC, et al. Valproic acid metabolites inhibit dihydrolipoyldehydrogenase activity leading to impaired 2- oxoglutarate-driven oxidative phosphorylation. Biochim Biophys Acta. 2007;1767(9):1126-1133.

21. Smirnova IN, Kistenev BA, Krotenkova MV, et al. Stroke-like course of mitochondrial encephalomyopathy (melas syndrome). Nervous diseases M Atmosphere . 2006;43-48.

22. Bindoff LA, Engelsen BA. Mitochondrial diseases and epilepsy. Epilepsia. 2012;53(Suppl 4):92-97. 